Die Fraktionen (ca. $15 \mathrm{ml}$ pro $30 \mathrm{~min}$ ) werden auf ihren Uronsäuregehalt getestet. Die einzelnen Fraktionen werden zusammengefaßt, gegen dest. Wasser chloridfrei dialysiert, gefriergetrocknet und ausgewogen. Die Aussagen über die Mucopolysaccharid-Peptide werden an Hand des Aminozuckerspektrums (Aminosäure-Analysator) erhalten.

Die vorliegenden Untersuchungen wurden in Zusammenarbeit mit Herrn Prof. Dr. Hauss und seinen Mit- arbeitern, Med. Klinik der Univ. Münster, durchgeführt, denen wir für die Bereitstellung des Aortenmaterials und für eingehende Diskussionen danken. Herrn Prof. Dr. Micheel sei für die Erlaubnis zur Benutzung des Aminosäuren-Analysators (Leihgabe des Landesamtes für Forschung des Landes NordrheinWestfalen) gedankt. - Dem Landesamt für Forschung des Landes Nordrhein-Westfalen und dem Verband der Chemischen Industrie „Fonds der Chemie" sind wir für die materielle Unterstützung zu Dank verpflichtet.

\title{
Zur Metachromasie von Toluidinblau in Gegenwart von Nucleinsäuren
}

\section{K. TEMPEL}

\author{
Institut für Pharmakologie, Toxikologie und Pharmazie der Tierärztlichen Fakultät \\ der Universität München (Vorstand: Prof. Dr. Dr. h. c. K. ZIPF) \\ (Z. Naturforschg. 24 b, 1279-1283 [1969] ; eingegangen am 23. April 1969)
}

1. Nach Zusatz von Nucleinsäuren zu wäßrigen Toluidinblaulösungen treten neue Farbqualitäten auf. Ihre Absorptionsmaxima lagen bei 655,585 und $555 \mathrm{~nm}$.

2. Art und Umfang dieser Metachromasie sind abhängig vom Reaktionsmilieu, vom Farbstoff-Polyanionen-Konzentrationsverhältnis sowie von Molekülgröße und Nativität der einzelnen Nucleinsäurepräparate.

3. In wäßrigen Proteinlösungen gelöste hochmolekulare DNS-Präparate verhalten sich gegenüber DNS-Lösungen ohne Proteinzusatz wie Präparate höherer Nativität.

4. Änderungen der metachromotropen Eigenschaften von DNS-Protein-Lösungen sind qualitativ und quantitativ als empfindlicher Nachweis für denaturierende Einflüsse (Hitze, ionisierende Strahlen, UV-Licht, Ultraschall, Tris- ( $\beta$-chloräthyl) -amin oder DNase I) verwertbar.

Das Wesen der Metachromasie besteht in der molekularen Wechselwirkung zwischen wäßrig gelösten kationischen Farbstoffen und anionischen Makromolekülen. Unter geeigneten Bedingungen kommt es zur genauen räumlichen Orientierung benachbarter Farbstoffmoleküle am Polyanion ${ }^{1-5}$. Daraus folgt, daß die metachromatische Reaktion qualitativ und quantitativ gewisse Rückschlüsse auf Art, Menge und sterische Anordnung der für die Reaktion verfügbaren anionischen Gruppen zuläßt ${ }^{6}$. Inwieweit dies auch für Nucleinsäuren gilt, wurde vorliegend am Beispiel der Metachromasie von Toluidinblau (TB) in Gegenwart verschiedener Desoxyribonucleinsäure (DNS) - und Ribonucleinsäure (RNS) -Präparate untersucht. Zur Denaturierung der DNS-Präparate dienten Hitze, Röntgenstrahlen, UVLicht, Ultraschall, Tris-( $\beta$-chloräthyl) -amin (Sinalost ${ }^{\circledR}$ ) und Desoxyribonuclease I (EC 3.1.4.5.) (DNase I).

1 M. Arnold, Histochemie 6, 1 [1966].

2 J.-P. Bidegaray et R. Viovy, J. Chim. physique 61, 1383 [1964].

3 J.-P. Bidegaray et R. Viovy, J. Chim. physique 61, 1391 [1964].

\section{Material und Methoden}

\section{M a terial}

1. Äthanol, 95-proz., mit Petroläther vergällt.

2. Albumin aus Hühnerei, Erg. B. 6, Riedel-DeHaen AG, Seelze.

3. Albumin aus Hühnerei, reinst, Serva, Nr. 11840.

4. Albumin aus Rinderserum, reinst, Serva, Nr. 11920.

5. Desoxyribonucleinsäure, Natriumsalz, aus Kalbsthymus, mind. 95\% DNS, MG $8900000, \mathrm{~N}$ ca. $14,5 \%$, P ca. $8,0 \%$, EGA-Chemie Keppler und Reif, Steinheim am Albuch (Präparat 1).

6. Desoxyribonucleinsäure aus Kalbsthymus, lyophilisiert, reinst, hochmolekular, rel. Viskosität ca. $3,0, t_{\mathrm{m}}$ mindestens $78{ }^{\circ} \mathrm{C}$, nach $\mathrm{Z}$ a m e $\mathrm{nh}$ of f, Serva Nr. 18560 (Präparat 2).

7. Desoxyribonucleinsäure aus Lachssperma, lyophilisiert, reinst, hochpolymer, praktisch protein- und salzfrei, $t_{\mathrm{m}}$ ca. $78{ }^{\circ} \mathrm{C}$, nach Emanuel und Ch ha i k of f, Serva Nr. 18570 (Präparat 3).

${ }^{4}$ R. Chayen and E. R. Roberts, Sci. J. Roy. Coll. Sci. 25, 50 [1955].

5 M. D. Schoenberg and R. D. Moore, Biochim. biophysica Acta [Amsterdam] 83, 42 [1964].

6 A. Krygier and K. Kasprzyk, Acta Med. Polona 2, 123 [1961]. 
8. Desoxyribonucleinsäure, Natriumsalz, nach $\mathrm{H}$ a m m a rsten, MG ca. 1-2 Millionen, reinst, C. Roth, Karlsruhe (Präparat 4).

9. Desoxyribonucleinsäure aus Salm, eiweißfrei, N ca. $15,2 \%, \mathrm{P}$ ca. $9,1-9,2 \%$, Feuchtigkeit $5,1 \%$, hochmolekular, rein, C. Roth, Karlsruhe (Präparat 5).

10. Desoxyribonucleinsäure, Natriumsalz aus Kalbsthymus, nach $\mathrm{Hamm}$ a r s e n, reinst, $\mathrm{MG}$ 1200000 , Fluka AG, Nr. 89380 (Präparat 6).

11. Desoxyribonucleinsäure aus Heringssamen, Dr. Th. Schuchardt GmbH, München, Hauptkatalog Nr. DE 054* (Präparat 7).

12. Desoxyribonucleinsäure, Natriumsalz, aus Heringssamen, Dr. Th. Schuchardt GmbH, München, Hauptkatalog Nr. DE 056 * (Präparat 8).

13. Desoxyribonuclease I (Desoxyribonucleat-oligonucleotidhydrolase, EC 3.1.4.5), lyophilisiert, rein, aus Rinderpankreas, 30000 Dornase-Einheiten pro mg, Serva Nr. 18550.

14. Diphenylamin p. A., Merck Nr. 3086.

15. Essigsäure, $99-100 \%$ (D ca. 1,05) p. A., Merck Nr. 90063.

16. Folin-Ciocalteus Phenolreagens, Merck Nr. 9001.

17. $\beta$-Globulin aus Rinderserum, lyophilisiert, rein, Serva Nr. 22490.

18. Kaliumacetat, reinst, Merck Nr. 4820.

19. Kaliumjodid p. A., Merck Nr. 5043.

20. Kupfersulfat, $\mathrm{CuSO}_{4} \cdot 5 \mathrm{H}_{2} \mathrm{O}$ p. A., Merck Nr. 2790.

21. Natriumcarbonat, wasserfrei p. A., Merck Nr. 6392.

22. Natronlauge, Titrisol ${ }^{\circledR}, 0,1-n .$, Merck Nr. 9959.

23. Ribonucleinsäure p. A., hochmolekular aus Hefe, $S_{20} \sim$ 8, Serva Nr. 34404 (Präparat 9).

24. Ribonucleinsäure pract., niedermolekular aus Hefe, Serva Nr. 34410 (Präparat 10).

25. Ribonucleinsäure (Hefenucleinsäure), Trockensubstanz $94 \%, \mathrm{~N} \geqq 15,3 \%, \mathrm{P} \geqq 8,7 \%$, Biuretreaktion: Negativ, Dr. Th. Schuchardt GmbH, München, Hauptkatalog Nr. Nu 87 (Präparat 11)*.

26. Ribonucleinsäure, Natriumsalz, Trockensubstanz $94 \%, \mathrm{~N} \geqq 14,2 \%, \mathrm{P} \geqq 8,4 \%$, Biuretreaktion: $\mathrm{Ne}-$ gativ, Dr. Th. Schuchardt GmbH, München, Hauptkatalog Nr. Nu 88 (Präparat 12)*.

27. Schwefelsäure, 95-97-proz. (D ca. 1,84) p. A., Merck Nr. 731.

28. Seignettesalz, K-Na-Tartrat p. A., Merck Nr. 8087.

29. Sinalost ${ }^{\circledR}$, Tris- $\left(\beta\right.$-chloräthyl) -amin $\cdot \mathrm{HCl}(\mathrm{HN} 3)^{*}$, Nordmark-Werke.

30. Toluidinblau O (TB), Merck Nr. 1273.

31. Trichloressigsäure (TCE) z. A., Merck Nr. 807.

* Den Firmen sei an dieser Stelle für die großzügige Úberlassung der Präparate gedankt.

7 W. C. Schneider, J. biol. Chemistry 161, 293 [1945].

8 W. J. Steele, N. Okamura, and H. Busch, Biochim. biophysica Acta [Amsterdam] 87, 490 [1964].
Met hodik

Zur Entwicklung der TB-Metachromasie wurden $0,5 \mathrm{ml}$ der wäßrigen Nucleinsäurelösungen mit $3 \mathrm{ml}$ einer wäßrigen, 1,67 mg-proz. TB-Lösung versetzt. 15 min später wurde die Farbdichte der Proben in 1,0-cm-Glasküvetten mit dem Spektralphotometer PMQ II der Fa. C. Zeiss gemessen. Zur Bestimmung der DNS-Konzentration der Proben wurde die Methode von SCHNEIDER $^{7}$ gering modifiziert: Die zur Fällung der DNS verwendete TCE-Endkonzentration betrug 4. Prozent. Die Präzipitate wurden zweimal mit einer 10-proz. äthanolischen Kaliumacetatlösung ${ }^{8}$ gewaschen.

Die Proteinkonzentrationen der Proben wurden mit der Biuretmethode ${ }^{9}$ oder nach LowRY et al. ${ }^{10}$ bestimmt.

\section{Denaturierende Einflüsse}

\section{a) Hitzeeinwirkung}

Wäßrige Nucleinsäurelösungen wurden $15 \mathrm{~min}$ im Wasserbad auf $90{ }^{\circ} \mathrm{C}$ erhitzt und unmittelbar anschließend mit Eiswasser auf $0{ }^{\circ} \mathrm{C}$ abgekühlt.

\section{b) Röntgenstrahlen}

Im allgemeinen wurden 1,56 bzw. $0,78 \mathrm{mg}$-proz. wäßrige DNS-Lösungen mit Oberflächendosen von $350 \mathrm{R}$ pro min unter sorgfältiger Kühlung röntgenbestrahlt (Kristalloflex 4 der Fa. Siemens und Halske, $60 \mathrm{kV}, 30 \mathrm{~mA}$, HWS $0,16 \mathrm{~mm} \mathrm{Cu}$, Filterung $0,16 \mathrm{~mm}$ $\mathrm{Cu}$, Abstand Focus - Objekt 10,0 cm) .

\section{c) UV-Licht}

Zur UV-Bestrahlung der Nucleinsäurelösungen diente die Analysenlampe S 500 der Quarzlampen-Gesellschaft $\mathrm{mbH}$, Hanau. Der Bestrahlungsabstand betrug $12,0 \mathrm{~cm}$, die Schichtdicke der Nucleinsäurelösungen $0,5 \mathrm{~cm}$. Die Proben wurden während der Bestrahlung gekühlt.

\section{d) Ultraschall}

Die Beschallung von Nucleinsäurelösungen wurde mit dem Branson-Sonifier, Modell S-125, HF-Generatur, Schallkopf mit Schallrüssel 1/2 durchgeführt. Im allgemeinen wurde - unter Kühlung - bei der höchsten mit diesem Gerät erreichbaren Energiestufe gearbeitet.

\section{e) Sinalost ${ }^{\circledR}$}

Im allgemeinen wurden 1,56 mg-proz. DNS-Lösungen $24 \mathrm{Stdn}$. bei Zimmertemperatur in Gegenwart von HN3-Konzentrationen $\geqq 2,48 \cdot 10^{-6} \mathrm{M}$ inkubiert.

9 G. Beisenherz, H. J. Boltze, T. Bücher, R. Czok, K. J. Garbade, E. Meyer-Ahrendt u. G. Pfleiderer, Z. Naturforschg. 8 b, 555 [1957].

10 O. H. Lowry, N. J. Rosebrough, A. L. Farr, and R. J. Randall, J. biol. Chemistry 193, 265 [1951]. 


\section{f) DNase I}

Zur Überprüfung der metachromatischen Aktivität enzymatisch abgebauter DNS wurde letztere unter gleichzeitigem Proteinzusatz in wäßriger Lösung zusammen mit DNase I (in Endkonzentrationen von $50-0,005 \mu \mathrm{g} / \mathrm{ml}$ ) ohne sonstige Zusätze bei $\mathrm{pH} 6,0$ und Zimertemperatur 30 min lang inkubiert.

\section{Ergebnisse}

Im sichtbaren Bereich hatte TB unter den gewählten Bedingungen ein Absorptionsmaximum bei $635 \mathrm{~nm}$ (Abb. 1). Nach Zusatz von Präparat 1 beispielsweise kam es zu negativer und positiver Metachromasie: Unter gleichzeitigem Extinktionsabfall

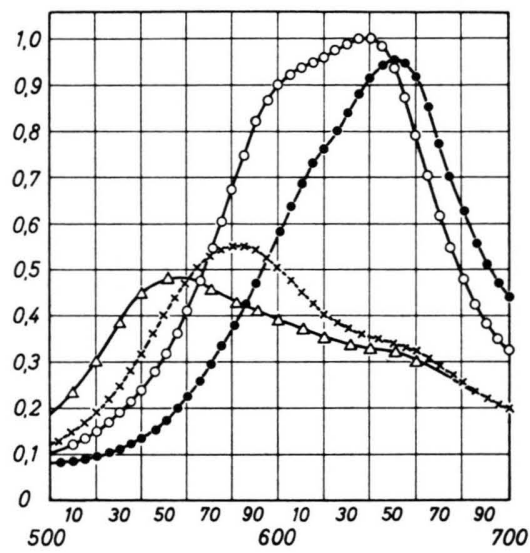

Abb. 1. Metachromasie von wäßrigen Toluidinblaulösungen in Gegenwart hochmolekularer DNS (Präparat 1). Abszisse: Wellenlänge in nm. Ordinate: Extinktionseinheiten. Alle Lösungen enthielten $14,29 \mu \mathrm{g} \mathrm{TB} / \mathrm{ml}$. 000 Ohne sonstige $\mathrm{Zu}$ sätze, $\cdots$ plus $89,6 \mu \mathrm{g}$ DNS/ml, +++ plus $11,2 \mu \mathrm{g}$ DNS/ $\mathrm{ml}, \triangle \triangle \triangle$ plus $5,6 \mu \mathrm{g} \mathrm{DNS} / \mathrm{ml}$.

bei $635 \mathrm{~nm}$ traten neue Farbqualitäten bei einem TB/DNS-Konzentrationsverhältnis von $0,16,1,28$, bzw. 2,55 auf, deren Absorptionsmaxima bei 655, 585 bzw. $555 \mathrm{~nm}$ lagen. Im jeweiligen Absorptionsmaximum verhielten sich die Maximalextinktionen bei $555,585,635$ bzw. $655 \mathrm{~nm}$ wie $1: 1,14: 2,08$ : 1,98 (Abb. 1).

Während die positive Metachromasie von TB bei $585 \mathrm{~nm}$ alle untersuchten Nucleinsäurepräparate umfaßte, trat sie bei $555 \mathrm{~nm}$ bevorzugt bei hochmolekularen DNS-Präparaten auf (Tab. 1 und 2). Dem Extinktionszuwachs bei dieser Wellenlänge war dabei ein Extinktionsabfall bei $585 \mathrm{~nm}$ korrelativ. Um Interferenzen des langwelligen Bereichs weitgehend auszuschließen, wurde den weiteren Aussagen der jeweils zwischen 530 und $570 \mathrm{~nm}$ erzielte Extinktionsunterschied zugrunde gelegt.
Der in Tab. 1 an Hand einiger Beispiele dargestellte prozentuale Bezug der bei $530 \mathrm{~nm}$ erzielten Extinktionswerte auf den zugehörigen Extinktionswert bei $570 \mathrm{~nm}$ in Abhängigkeit von der Nucleinsäurekonzentration belegt den relativen Extinktionsabfall bei der kürzeren Wellenlänge proportional zur Verkleinerung des Moleküls. Je kleiner andererseits die relative Extinktion bei $530 \mathrm{~nm}$, um so geringer der beiderseitige Extinktionszuwachs (bzw. -abfall) in Abhängigkeit suboptimaler Polyanionenzusätze (Tab. 1).

\begin{tabular}{lrrrrrr}
\hline $\begin{array}{l}\text { Prä- } \\
\text { parat }\end{array}$ & \multicolumn{5}{c}{ Nucleinsäurekonzentration in $\mu \mathrm{g} / \mathrm{ml}$} \\
& 1,4 & 2,8 & 5,6 & 11,2 & 22,4 & 44,8 \\
\hline 1 & 55,4 & 70,1 & 80,6 & 66,1 & 52,5 & 35,8 \\
11 & 45,6 & 47,7 & 48,2 & 44,5 & 41,4 & 40,5 \\
9 & 43,8 & 51,1 & 59,3 & 50,9 & 42,9 & 40,6 \\
$1^{*}$ & 45,0 & 46,5 & 49,0 & 46,6 & 40,5 & 36,8 \\
$1^{* *}$ & 51,4 & 61,2 & 68,0 & 56,5 & 43,4 & - \\
$1 * * *$ & 43,9 & 42,2 & 43,3 & 43,7 & 42,4 & 40,4 \\
\hline
\end{tabular}

Tab. 1. Relative Metachromasie von TB bei $530 \mathrm{~nm}$ in Gegenwart von Nucleinsäuren. Alle Angaben beziehen sich prozentual auf den bei $570 \mathrm{~nm}$ gemessenen Extinktionswert $(=100 \%)$. * Präparat 1 hitzedenaturiert, ${ }^{* *}-$ nach Röntgenbestrahlung mit $21,0 \mathrm{kR},{ }^{* * *}-$ in Gegenwart von $6,2 \cdot 10^{-4} \mathrm{M}$ HN3.

Analog verhielten sich denaturierte DNS-Präparate (Tab. 1).

Besonders kleine Extinktionsdifferenzen erbrachten die Präparate 1, 4 und 6. Der daraus ableitbare Schluß auf einen gegenüber vergleichbaren DNSPräparaten höheren Ordnungszustand wird durch den bei ersteren stärkeren Zuwachs der Extinktionsdifferenz zwischen den beiden Wellenlängen nach Hitzedenaturierung (Tab. 2) gestützt.

Die zur Erzeugung der bei $555 \mathrm{~nm}$ maximal absorbierenden positiven Metachromasie - verglichen mit den übrigen Präparaten - hohe Einwaage der Präparate 4 und 6 verweist auf Nucleinsäure-fremde Beimengungen. Tatsächlich erbrachte die DNS-Bestimmung mit der Dische-Reaktion unter Voraussetzung identischer Einwaagen nur 59\% der DNSKonzentration von Präparat 1 . Der Proteingehalt der $10 \mathrm{mg}$-proz. Lösungen verhielt sich wie $10: 1$. Während alle untersuchten Nucleinsäurepräparate Licht der Wellenlängen 230, 260 und $280 \mathrm{~nm}$ wie $(0,470 \pm 0,037): 1,0:(0,600 \pm 0,022)$ absorbierten, lautete das gleiche Extinktionsverhältnis für die Präparate 4 und 6: $0,950: 1,0: 0,770$ und bestätigt damit ihren höheren Proteingehalt. 


\begin{tabular}{|c|c|c|c|c|c|c|c|}
\hline \multirow{2}{*}{$\frac{\text { Präparat }}{\text { Nr. }}$} & \multirow[t]{2}{*}{ I } & \multicolumn{2}{|c|}{ II } & \multicolumn{2}{|c|}{ III } & \multicolumn{2}{|c|}{ IV } \\
\hline & & A & B & A & B & $\mathrm{A}$ & B \\
\hline 1 & 4,2 & 61,6 & 85,8 & 0,156 & 0,066 & 65,6 & 410,6 \\
\hline 2 & 4,2 & 56,0 & 80,8 & 0,185 & 0,090 & 14,0 & 226,7 \\
\hline 5 & 4,2 & 59,5 & 76,5 & 0,200 & 0,115 & 30,8 & 147,8 \\
\hline 3 & 4,2 & 61,4 & 81,9 & 0,201 & 0,092 & 39,3 & 247,8 \\
\hline 6 & 11,2 & 81,4 & 82,1 & 0,071 & 0,068 & 184,0 & 191,0 \\
\hline 4 & 11,2 & 74,6 & 75,1 & 0,114 & 0,097 & 111,0 & 160,4 \\
\hline 7 & 4,2 & 51,0 & 52.9 & 0.254 & 0.241 & 4,7 & 6,6 \\
\hline 8 & 2,8 & 52,3 & 52,4 & 0,235 & 0,239 & 3,5 & 2,0 \\
\hline 9 & 2,8 & 61,1 & 60,7 & 0,147 & 0,211 & 4,8 & 4,3 \\
\hline 12 & 2,8 & 60.9 & 60,2 & 0,188 & 0,198 & 11,1 & 11,1 \\
\hline
\end{tabular}

Tab. 2. Metachromasie von TB in Gegenwart von Nucleinsäuren bei 530 und $570 \mathrm{~nm}$. TB-Konzentration $14,29 \mu \mathrm{g} / \mathrm{ml}$ Ansatz. Spalte I: Nucleinsäurekonzentration (in $\mu \mathrm{g} / \mathrm{ml}$ ) unter den Bedingungen maximaler Metachromasie bei $530 \mathrm{~nm}$. Spalte II: Maximalextinktion bei $530 \mathrm{~nm}$ in Prozent, bezogen auf den zugehörigen Extinktionswert bei $570 \mathrm{~nm}$. Spalte III: Extinktionsdifferenz zwischen 570 und $530 \mathrm{~nm}$. Spalte IV:Zuwachs der Extinktionsdifferenz zwischen 570 und $530 \mathrm{~nm}$ nach Hitzedenaturierung des jeweiligen Nucleinsäure-Präparates in Prozent. Spalten A: Nucleinsäuren in wäßriger Lösung ohne sonstige Zusätze. Spalten B: Nucleinsäuren nach Lösung in $20 \mathrm{mg}$-proz. Ovalbuminlösungen.

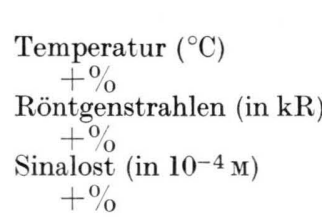

Temperatur $\left[{ }^{\circ} \mathrm{C}\right]$ $+\%$

Röntgenstrahlen (in $\mathrm{kR}$ ) $+\%$

Ultraschall (Beschallungsdauer in $\mathrm{min}$ ) $+\%$

UV-Licht (Bestrahlungszeit in min) $+\%$

Sinalost (in $\left.10^{-4} \mathrm{M}\right)$ $+\%$

DNase I (in $\mu \mathrm{g} / \mathrm{ml}$ Ansatz) $+\%$
DNS ohne Proteinzusatz

$\begin{array}{cccc}45 & 60 & 75 & \\ 55 & 148 & 226 & \\ 5,25 & 10,5 & 21,0 & 42,0 \\ 20,5 & 37,3 & 92,8 & 155,4 \\ 2,48 & 4,96 & 9,92 & 19,84 \\ 95,5 & 144,9 & 177,5 & 194,4\end{array}$

DNS in Gegenwart von Albumin oder Globulin (20 mg- $\%$ )

$\begin{array}{rrrrrrrr}20 & 30 & 40 & 50 & 60 & 70 & 80 & 90 \\ - & 85 & 201 & 261 & 321 & 342 & 342 & 349\end{array}$

$0,88 \quad 1,75$

7,7

24,6

0,5

66,1

15

6,9

1,24

156,5

0,005

3,10

6,3

243,4

0,05

15,8
3,50

44,6

1,0

91,4

30

17,2

6,20

298,9

$\begin{array}{rrr}0,5 & 5,0 & 50,0 \\ 62,1 & 186,3 & 218,9\end{array}$

Tab. 3. Denaturierende Einflüsse in ihrer Wirkung auf die metachromotropen Eigenschaften eines hochmolekularen DNSPräparates (Präparat 1). TB-Konzentration: 14,29 $\mu \mathrm{g} / \mathrm{ml}$, DNS-Konzentration 5,6 $\mu \mathrm{g} / \mathrm{ml}$. Angaben des prozentualen Zuwachses der Extinktionsdifferenz zwischen 570 und $530 \mathrm{~nm}$, bezogen auf die nicht-denaturierte Kontrolle. Jeder Wert stellt das Mittel zweier Doppelbestimmungen dar. Durchschnittlicher Variationskoeffizient aller Meßreihen 6,3 (4,9-6,8) Prozent.

Vor allem der letztere Befund veranlaßte aber zu der Annahme, daß Lösung hochmolekularer DNS in Gegenwart von Protein zu metachromotropen Präparaten mit dem Erscheinungsbild höherer Nativität führt. Diese Annahme wird gestützt 1. durch den Abfall der Extinktionsdifferenzen zwischen 570 und $530 \mathrm{~nm}$ bei Lösung hochmolekularer DNS in Gegenwart von Protein, 2. das Ausbleiben dieser Erscheinung bei DNS-Präparaten kleinen Mol.-Gew. und RNS und 3. den starken Temperatureffekt bei DNS hohen Mol.-Gew. und sein Ausbleiben bei kleinmolekularen DNS-Präparaten und der RNS (Tab. 3). Das gleiche gilt sinngemäß für denaturierende Einflüsse anderer Art (ibid.) .
Die das Auftreten von Metachromasie bei $555 \mathrm{~nm}$ begünstigende Wirkung zeigten Albumin- und Globulinpräparate qualitativ gleichwertig. Unterschiedlich waren die dafür optimalen Proteinkonzentrationen (Tab. 4).

\begin{tabular}{lcccc}
\hline Protein & & & & \\
\hline $\begin{array}{c}\text { [mg-proz. } \\
\text { Ovalbumin } \\
\text { (Präp. 2) }\end{array}$ & 0,063 & 0,066 & 0,085 & 0,166 \\
$\begin{array}{c}\text { Ovalbumin } \\
\text { (Präp. 3) }\end{array}$ & 0,101 & 0,161 & 0,190 & 0,210 \\
$\beta$-Globulin & 0,079 & 0,093 & 0,137 & 0,180 \\
\hline
\end{tabular}

Tab. 4. Extinktionsdifferenz $570-530 \mathrm{~nm}$ einer wäßrigen TB-Lösung in Gegenwart von Präparat 1 und Protein in Abhängigkeit von der Proteinkonzentration. TB-Konzentration: $14,29 \mu \mathrm{g} / \mathrm{ml}$, DNS-Konzentration: $5,6 \mu \mathrm{g} / \mathrm{ml}$. 
Hitzedenaturierte Proteine waren praktisch gleich wirksam. Nachträglicher Zusatz von Protein zu wäßrigen DNS-Lösungen blieb ohne Einfluß.

Auch der Umfang der „Denaturierung“ hängt von der Proteinkonzentration ab: Wurde beispielsweise wäßrig gelöste DNS (Präparat 1, 5,6 $\mu \mathrm{g} / \mathrm{ml}$ ) in Gegenwart von $\beta$-Globulin entsprechend Endkonzentrationen von $40,20,10,5$ bzw. 2,5 mg-proz. mit $12 \mathrm{kR}$ röntgenbestrahlt, vergrößerte sich die Extinktionsdifferenz zwischen 570 und $530 \mathrm{~nm}$ um 87,5, 149,3, 125,0, 74,1 bzw. 51,5 Prozent.

\section{Diskussion}

$\mathrm{Zu}$ den wesentlichsten Voraussetzungen für das Auftreten von Metachromasie gehört die genaue räumliche Orientierung kationischer Farbstoffmoleküle an der polyanionischen Oberfläche metachromotropen Materials ${ }^{1-5}$. „Le colorant se comporte come s'il était polymérisé" 3 . An der Ausbildung solcher Wechselwirkungen sind $u$. a. polare Bindungen und Van der W a als-Kräfte beteiligt ${ }^{1-5}$. Das Reaktionsmilieu ist mithin von ausschlaggebender Bedeutung ${ }^{1-6}$. Als Lösungsmittel für metachromatische Färbungen wird dest. Wasser bevorzugt, da es die Dissoziation von kationischen und anionischen Gruppen ermöglicht ${ }^{1}$.

11 F. I. Braginskaya i I. E. Elpiner, Biofizika 9, 40 [1964].

12 Y. Fuse, K. Miyaki, and M. Tsuboi, Bull. chem. Soc. Japan 38, 1039 [1965].

13 M. E. Lamm, L. Childers, and M. K. Wolf, J. Cell Biol. 27, 313 [1965].

14 A. Miura and Y. Ohba, Biochim. biophysica Acta [Amsterdam] 145, 436 [1967].

15 M. Semmel et J. Huppert, C. R. hebdom. Séances Acad. Sci. 254, 3746 [1962].
Neben dem Reaktionsmilieu ist das Konzentrationsverhältnis Farbstoff : Polyanion entscheidend. Je höher dieser Quotient, um so höher im allgemeinen der „metachromatische Index“ (l. c. 1-6,11-14 sowie vorliegende Befunde). Bestimmte „Polymerisationsgrade" treten dabei bevorzugt in Erscheinung (ibid., vorliegende Befunde).

Ändern sich bei Konstanz des Reaktionsmilieus - etwa unter dem Einfluß physikalischer Noxen (l. c. ${ }^{11,15-17}$, vorliegende Befunde) - die metachromotropen Eigenschaften eines Polyanions, so ist dies als Ausdruck von Änderungen seiner Molekülstruktur zu werten (l. c. ${ }^{5,11,15,16}$ u. a.). Die vorliegenden Ergebnisse sprechen dafür, daß die bei 555 nm auftretende metachromatische Qualität von TB in Gegenwart von Nucleinsäuren als Funktion räumlich geordneter, wahrscheinlich helikaler Strukturen aufgefaßt werden kann. Berücksichtigt man, daß unter den gewählten Versuchsbedingungen beispielsweise bereits Röntgenstrahlendosen im Bereich der $\mathrm{DL}_{50 / 30}$ der Maus und darunter ${ }^{18}$ nachgewiesen werden konnten, so ergibt sich zudem - auch im Vergleich mit analogen In vitro-Systemen ${ }^{19-21}$ - die besondere Empfindlichkeit der Versuchsanordnung. Da Proteinzusatz die Metachromasie von TB in Gegenwart hochmolekularer DNS bei $555 \mathrm{~nm}$ erhöht, folgt, daß dieses System in seiner metachromotropen Eigenschaft gegenüber TB als Annäherung an ein natives DNS-Protein-Modell gewertet werden kann.

16 M. Semmel et J. Huppert, ibid. 256, 5649 [1963].

17 M. Semmel et J. Huppert, Arch. Biochem. Biophysics 108, 158 [1964].

18 K. Tempel, Zbl. Veterinärmed., Erg.-Band 8.

19 M. Vogt u. E. Harbers, Strahlentherapie 133, 426 [1967].

20 H. Harrington, J. molecular Biol. 15, 152 [1966].

21 H. Weinert u. U. Hagen, Strahlentherapie 136, 204 [1968]. 